\title{
Differential electron-capture cross-sections in high energy ion-atom collisions : comparison of experiment and theory for the Thomas peak
}

\author{
R. D. Rivarola
}

Instituto de Física Rosario (CONICET-UNR), Pellegrini 250, 2000 Rosario, Argentina

\author{
A. Salin \\ Laboratoire des Collisions Atomiques (*), Université de Bordeaux I, 33405 Talence, France \\ and M. P. Stockli
}

J. R. Macdonald Laboratory, Physics Department, Kansas State University, Manhattan, Kansas 66506, U.S.A.

(Reçu le 12 décembre 1983, accepté le 19 janvier 1984)

\begin{abstract}
Résumé. - On compare l'observation expérimentale récente du " pic de Thomas » dans la section différentielle de capture d'électrons à haute énergie avec les prédictions théoriques. Un très bon accord est obtenu à tous les angles de diffusion avec l'approximation CDW tenant compte de l'interaction entre les noyaux.

Abstract. - The recent experimental observation of the Thomas peak on the differential crosssection for high energy electron capture is confronted with the theoretical prediction. Very good agreement is obtained for all scattering angles with the $\mathrm{CDW}$ approximation including the internuclear interaction.
\end{abstract}

The existence of a peak in the differential cross-section for electron capture in high energy ion-atom collisions is one of the most striking theoretical prediction showing that first order perturbation theory cannot describe adequately high energy rearrangement processes. This peak has been named the " Thomas peak » since Thomas in 1927, using classical arguments, proposed a two-step mechanism to explain high energy charge exchange [1]. His contribution was based on the observation that, if the target electron is considered to be at rest with respect to the target, it cannot be captured in a single binary interaction with the projectile because of energy and momentum conservation laws. Hence he proposed his two-step process where the electron interacts first with the projectile then with the target nucleus. It has been well established by many authors

(*) Equipe de Recherche CNRS no 260. 
(see references in [2] and [3]) that this simple picture has a quantal analog. However the Thomas process dominates only at very high energies $(>100 \mathrm{MeV} / \mathrm{amu})$ for the evaluation of total capture cross-sections, unless one considers transitions between excited states [2]. In fact, it is overshadowed in total cross-sections by the contribution due to the electronic momentum distribution in the initial state (i.e. the electron cannot be considered at rest in the target frame). For that reason, measurements on differential cross-sections at high energies were required to show up the Thomas two-step process and this has been achieved by Horsdal-Pedersen et al. [4].

In the present work we make a comparison between the experimental and theoretical results on the differential cross-section for electron capture by protons in helium and hydrogen. We use the CDW approximation [5] which is well documented so that it is not necessary to reproduce here details of its derivation $[3,6]$.

In order to compare the calculated cross-sections with the experimental data one has to take into account that the experimental scattering angle had a finite resolution dominated by the finite slit openings and small angle scattering in the residual and target gas. Hence the angular resolution function was determined without changing the target gas pressure by measuring the one dimensional profile to the direct beam hitting the position sensitive detector. In the helium experiment of [4] it was reported that these measured profiles were fitted with the sum of two concentric Gaussians. A more accurate beam profile measurement was performed in the $\mathrm{H}_{2}$ experiment [4] and a detailed analysis showed that the linear combination of three concentric Gaussians gives a significantly improved representation of the beam profile for the $\mathrm{H}_{2}$ (Fig. 1) as well as for all the He experiments. Especially the far wings of the profiles are much better reproduced. Despite the fact that they are two to four order of magnitude smaller than the central part, they are important for an accurate convolution, since the measured cross-section varies up to four orders of magnitude in a single experiment. The resolution function was assumed to have the form

$$
P(\theta)=\sum_{i=1}^{3} \frac{a_{i}}{\sqrt{2 \pi} \sigma_{i}} \exp \left(-\frac{\theta^{2}}{2 \sigma_{i}^{2}}\right) \text { where } \sum_{i=1}^{3} a_{i}=1
$$

and $\theta$ is the scattering angle in the laboratory frame. Table I shows the values of the fitted para-

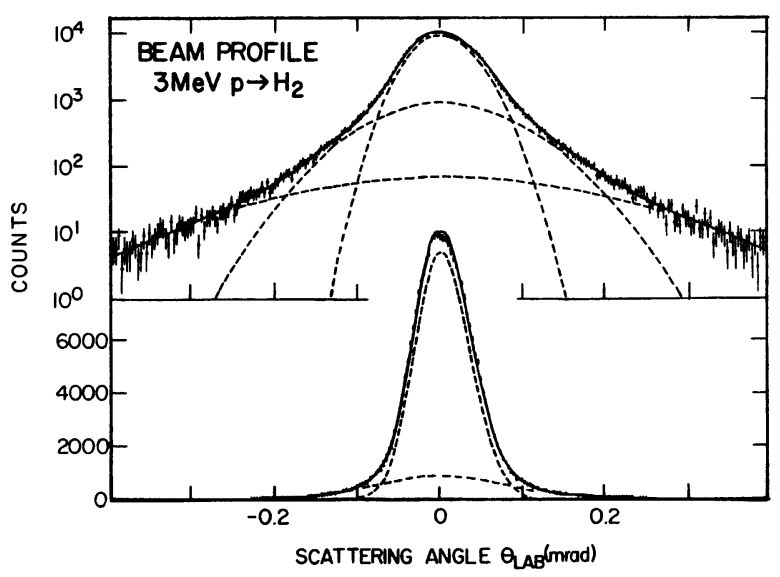

Fig. 1. - Logarithmic and linear profiles of the direct $3 \mathrm{MeV}$ proton beam $5.7 \mathrm{~m}$ after passing the gas cell which is $0.27 \mathrm{~m}$ long and was filled with 72 mtorr hydrogen gas. The full curve shows the sum of the three concentric Gaussian functions, which fits the measured profile almost perfectly over $31 / 2$ orders of magnitude. The dashed curves show the individual Gaussian functions. In the lower linear display the least intense Gaussian is too small to be plotted. 
Table I. - Beam profile parameters.

\begin{tabular}{lccc} 
& $\# 1$ & $\# 2$ & $\# 3$ \\
\hline p-He & $23(1)$ & $66(3)$ & $150(8)$ \\
$2.82 \mathrm{MeV}$ & $74(1)$ & $21(1)$ & $5(1)$ \\
\hline $\mathrm{p}-\mathrm{He}$ & $25(1)$ & $61(3)$ & $154(11)$ \\
$5.42 \mathrm{MeV}$ & $59(3)$ & $32(2)$ & $9(2)$ \\
\hline $\mathrm{p}-\mathrm{He}$ & $26(1)$ & $63(5)$ & $158(30)$ \\
$7.40 \mathrm{MeV}$ & $71(3)$ & $25(2)$ & $4(1)$ \\
\hline $\mathrm{p}-\mathrm{H}_{2}$ & $33(1)$ & $76(2)$ & $170(9)$ \\
$3.0 \mathrm{MeV}$ & $80(1)$ & $17(1)$ & $3(0.4)$ \\
\hline
\end{tabular}

The beam profiles of all discussed experiments [4] were fitted with a linear combination of three concentric Gaussians. The upper number gives the width (sigma) $\sigma_{i}$ in units of $10^{-6}$ radian and the lower number gives the fraction $a_{i}$ in percent. The errors given in parentheses represent only the uncertainties of the fitted beam profiles. Errors from elliptical components or time variations of the beam profile are expected to be quite small.

meters of all the profiles. One observes that most profiles are quite similar except in the $\mathrm{H}_{2}$ experiment where the profile has a wider central part because the beam was less collimated in order to compete with the smaller capture cross section [4].

A deconvolution of the measured data gives rise to huge relative uncertainties in the range of small cross sections (valleys) induced by the large statistical scatter of the data and the uncertainty of the beam profile. In order to reduce this problem the calculated differential cross-sections $\left(f(\theta)=.(\mathrm{d} \sigma / \mathrm{d} \Omega)_{\text {lab }}\right)$ were convoluted with the corresponding beam profile $P(\theta)$ :

$$
f^{*}\left(\theta_{0}\right)=2 \int_{\theta=0}^{\infty} \int_{\phi=0}^{\pi} f(\theta) \cdot P\left(\sqrt{\left.\theta_{0}^{2}+\theta^{2}-2 \cdot \theta_{0} \theta \cdot \cos \phi\right)} \cdot \theta \cdot \mathrm{d} \phi \cdot \mathrm{d} \theta\right.
$$

The convoluted function $f^{*}\left(\theta_{0}\right)$ is shown in figures 2 and 3. However detailed comparison with the measured data requires an analysis of the errors introduced with the convolution. For this purpose the convolution was repeated with modified profiles, with a similar but less accurate representation of the measured beam profile (for example the earlier published profiles in [4]). The obtained differences of the convoluted functions were used as an error estimate. In most cases these deviations are quite small and hardly exceed the $5 \%$ level. However the range of 0.25 to $0.40 \mathrm{mrad}$, where the valley is filled in, is more sensitive and shows uncertainties less then $10 \%$ for the $\mathrm{H}_{2}$ experiment but as much as $20 \%$ for the He experiment, as the latter profile is not so accurately known.

\section{Proton-helium collisions.}

We use for the helium wave-function the analytic Hartree-Fock wave function from table I of Clementi and Roetti [7]. The rôle of electron correlation in the initial state has been discussed in [8], however such a study has not been carried out on differential cross-sections. Our calculations include the internuclear potential, in contrast with other approaches to date, which plays an important rôle for angular scattering, particularly for angles larger than that of the Thomas peak. 


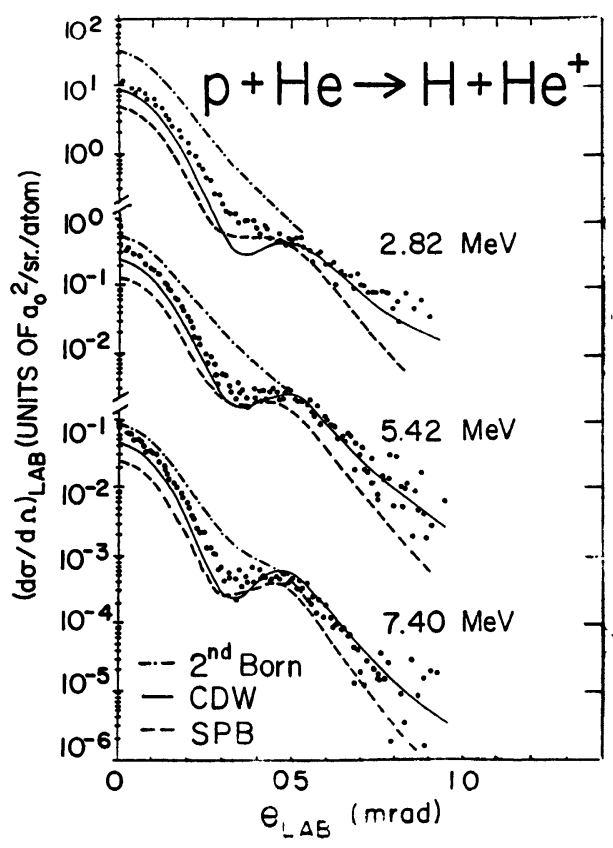

Fig. 2. - Differential electron-capture cross-sections by protons from helium gas. Experimental data [4] are represented as dots. Theoretical data convoluted with appropriate beam profile. Full curve : present CDW results. Dashed curve : SPB [9]. Dot-dash curve : Second Born [13].

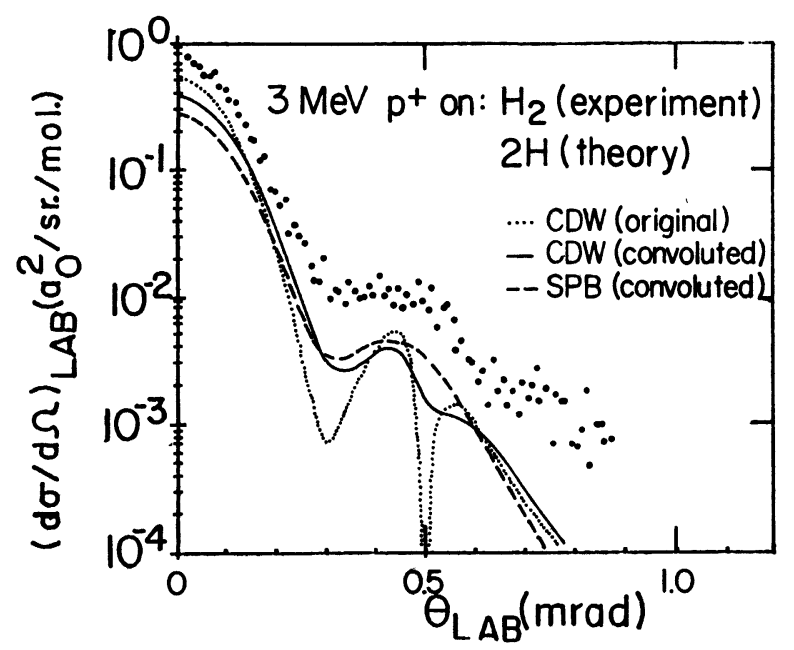

Fig. 3. - Differential electron-capture cross-sections by protons from hydrogen. Experimental data [4] on $\mathrm{H}_{2}$ are represented as dots. Theory : dotted curve shows the original CDW calculations whereas the full curve (present CDW results) and dashed curve (SPB [9]) results from a convolution with the appropriate beam profile. All theories are relative to two hydrogen atoms. 
Our results are given in figure 2 for various energies. Clearly, a very good agreement is obtained both around the Thomas peak $(\theta \sim 0.45 \mathrm{mrad})$ and for small angles of scattering. We also give in the same figure results obtained with the Strong Potential Born approximation [9] and the Second Born Approximation [13]. Both approximations do not agree with the data as well as the CDW approximation, especially for small scattering angles which determine the total cross-section. This disagreement is not due to the neglect of the internuclear potential in the latter calculations. We do not give here the results of the Strong Potential Born approximation obtained through a peaking approximation [10] since the latter introduces a large error, as shown by [9].

\section{Proton-hydrogen collisions.}

We have carried out similar calculations on atomic hydrogen. However, experimental results are for molecular hydrogen. We give in figure 3 a comparison between theory and experiment based on the often used approximation that $\mathrm{H}_{2}$ is equivalent for this process to two independent $\mathrm{H}$ atoms. Clearly the agreement is not as good as in the previous case, particularly the absolute magnitude of the Thomas peak. Further studies are necessary to check whether our crude treatment of the hydrogen molecule could be the source of this discrepancy. However, for small scattering angles the discrepancy can be compared with the one found for total cross-sections in the same energy range (see [3], Fig. 7). Attention should be drawn to the structure in the theoretical CDW data which is superposed on the Thomas peak [11]. The experimental resolution does not wash out this structure which appears as an undulation at $0.5 \mathrm{mrad}$ as shown in figure 3 . Such a structure is not predicted by any other theory, which provides an invaluable test for the CDW theory. Despite some undulations one can see in the experimental data, the statistical scatter is too large to allow a significant test for the existence of such a structure. It was already pointed out in [6] that such a structure cannot be observed for the capture from $\mathrm{He}$.

As a conclusion, the experimental data seem to fit quite well the theoretical predictions in the case of helium, for the Thomas process as well as for small scattering angles which determine the total cross-section. On the other hand, the experiments on $\mathrm{H}_{2}$ cannot still confirm the prediction of a structure superimposed on the Thomas peak [11]. The present test, following a very large class of previous ones (e.g. $[3,6,12])$, shows that the CDW approximation is, in contrast with all existing alternative approaches, as reliable for high energy electron capture as the first Born approximation for excitation.

\section{Acknowledgments.}

Thanks are due to Pr. J. H. McGuire for communication of results in advance of publication.

Martin P. Stockli was supported by the U.S. Department of Energy, Division of Chemical Sciences.

\section{References}

[1] Thomas, L. H., Proc. R. Soc. A 114 (1927) 561-76.

[2] Shakeshaft, R. and Spruch, L., Rev. Mod. Phys. 51 (1979) 369-405.

[3] Belkić, Dž., Gayet, R. and Salin, A., Phys. Rep. 56 (1979) 279-369.

[4] Horsdal-Pedersen, E., Cocke, C. L. and Stockli, M., Phys. Rev. Lett. 50 (1983) 1910-3. Horsdal-Pedersen, E., Cocke, C. L. and Stockli, M., Proceedings of the Thirteenth International Conference on the Physics of Electronic and Atomic Collisions (Berlin, 1983).

[5] Cheshire, I. M., Proc. Phys. Soc. 84 (1964) 89-98.

[6] Rivarola, R. D. and Salin, A., J. Phys. B, in press.

[7] Clementi, E. and Roetti, C., Atomic Data 14 (1974) 177-478. 
[8] Banyard, K. E. and Szuster, B. J., Phys. Rev. A 16 (1977) 129-132.

[9] McGuire, J. H. and Sil, N. C., Private communication.

[10] Macek, J. H. and Alston, S., Phys. Rev. A 26 (1982) 250.

[11] Rivarola, R. D. and Miraglia, J. E., J. Phys. B 15 (1982) 2221-32.

[12] Chetioui, A., Wohrer, K., Rozet, J. P., Jolly, A., Stephan, C., Belkić, Dž., Gayet, R. and Salin, A., J. Phys. B 16 3993-4003.

Andriamonje, S., Chemin, J. F., Roturier, J., Saboya, B., Scheurer, J. N., Gayet, R., Salin, A., Laurent, H., Aguer, P. and Thibaud, J. P., soumis à $Z$. Phys.

Rozet, J. P., Lagagneux-Piquemal, P., Chetioui, A. et Chevallier, P., Proceedings of the Thirteenth International Conference on the Physics of Electronic and Atomic Collisions (Berlin, 1983).

[13] Simony, P. R., McGuire, J. H. and Eichler, J., Phys. Rev. A 26 (1982) 1337-1343. 